\title{
Optimization of a ship's ICCP system to minimize electrical and magnetic signature by mathematical simulation
}

\author{
S. Xing ${ }^{1,2}$, J. Wu ${ }^{1,2} \&$ Y. Yan ${ }^{1,2}$ \\ ${ }^{I}$ State Key Laboratory for Marine Corrosion and Protection, P.R. China \\ ${ }^{2}$ Luoyang Ship Material Research Institute, P.R. China
}

\begin{abstract}
In this paper, the protective effect and distribution of the magnetic field of cathodic protection (CP) systems designed by experience were calculated by the boundary element method (BEM), and the calculation results had agreement with the measured results when the ship berthed at port, which suggested that calculation results are accurate. The performance of the $\mathrm{CP}$ system was optimized by moving the position of the anodes, as well as changing the output current of the anodes. The objective was to design the system to minimize the electric and magnetic field and provide adequate protection. Calculation results show that when the anodes were symmetrically installed at the $130^{\text {th }}$ frame and the $232^{\text {nd }}$ frame, the corrosion related magnetic field and total output current were reduced by about $40 \%$ and $8.6 \%$ respectively.

Keywords: cathodic protection, mathematical simulation, boundary element method, electric and magnetic field.
\end{abstract}

\section{Introduction}

Coatings combined with impressed current cathodic protection (ICCP) are the most common means for the protection of shipboard. They interact with each other to protect shipboard. Coatings provide primary corrosion protection by isolating the hull metal from the seawater, while ICCP provides secondary corrosion protection in those areas where the paint is damaged or degraded. The protective effect is directly related to the ICCP configurations; an incorrectly designed ICCP system would not only influence the protective effect but also 
influence the distribution of Underwater Electrical Potential (UEP) and corrosion related magnetic (CRM), which are known as the signature of the ship.

Electric fields and coincident magnetic fields arise around a ship due to the current flow from the ICCP system. UEP and CRM signatures exist even in the absence of a cathodic protection (CP) system. They are caused by the galvanic potential differences between the metallic structures in contact with the seawater. For example, the hull and propeller provide sufficient driving potential to create an electric field. These electromagnetic fields take the form of steady electric and steady magnetic fields arising from the steady flow of current around the hull of the vessel. Modern mines can detect these fields and use them to detect and classify passing ships.

Researches [1-4] suggest that the design and optimization of the ICCP system is an effective means of preserving the integrity of a vessel and controlling the signatures. The design of an optimized ICCP system requires accurate placement of the anodes and reference electrodes on the ship's hull. Many shipboard ICCP systems currently in use were designed using relationships and engineering judgment. However, the protection effect of the CP system designed by experience cannot be known and sometimes it might lead to under or over protection and a high electric signature. Today, computer simulation techniques based on BEM, such as BEASY, have enabled the electric and magnetic field generated by the galvanic interaction of the ship's metallic structure and ICCP system to be predicted. Thus, it provides a tool to predict changes in the protection level of the ship and the electric field in the seawater caused by the ICCP system

In this paper, BEASY was used to predict and optimize the distribution of the potential and electric field signatures.

\section{Experimental}

\subsection{Model}

A vessel with a length of 125 meters was investigated. The ship had 250 frames with an interval of $0.5 \mathrm{~m}$ and the frames were assigned 0 to 250 from bow to stern. The geometry of interest in the boundary element model was the wetted surface of the hull and major appendages. The ship has two propellers and two rudders, and the propellers were made of nickel-aluminium-bronze alloy (NAB) and modelled as solid disks with a surface area equivalent to the real propellers. The shaft is made of carbon steel and the propellers and shafts were assumed to be uncoated because of turbulence engendered by propeller movement. The ship's hull and rudders are also made of carbon steel, which were coated to prevent corrosion. The ICCP system evaluated included four anodes and a centre controlled power supply. The half of ship BEM model was shown in Figure 1.

\subsection{Boundary condition}

The cathodic polarization curves of uncoated and coated carbon steel were shown in figure 2(a) and the polarization curve of NAB was shown in figure 


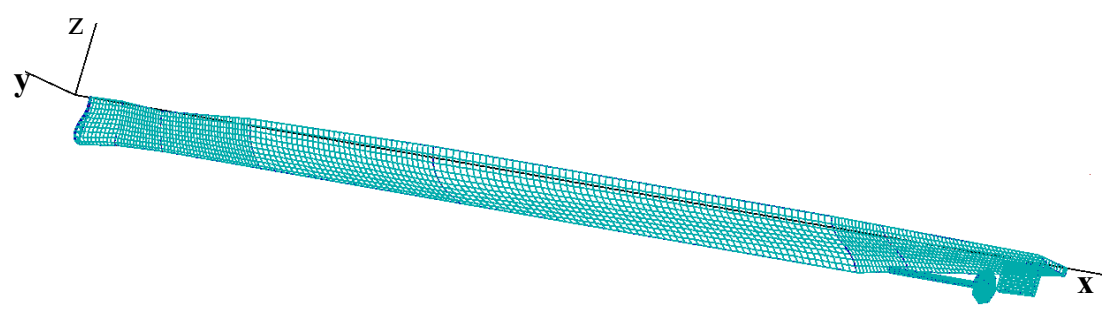

Figure 1: $\quad$ BEM model of the ship.

2(b), which was set as the boundary condition of the ship model. During calculation, the polarization curve of coated steel was set as the boundary condition of the ship's hull and rudder, the polarization curve of uncoated steel was set as the boundary condition of the shafts and the polarization curve of NAB was set as the boundary condition of the propellers. A box of 2500 meters was created as the infinite boundary where the current density was zero.

\subsection{ICCP optimization}

The optimization process required the problem to be posed in the formed of an objective function, designed variables and constraints. The least squares of the potential on the element of the surfaces with respect to the potential target value of the surfaces were used as an objective function to smooth the potential $[5,6]$.

$$
o b j=\sum_{i=1}^{n_{s}} \sum_{j=1}^{n_{e}}\left(\phi_{i j}-\phi_{t \arg e t, i}\right)^{2}
$$

Subject to the following constraints on the surface of the cathode [3]

$$
\begin{gathered}
g_{i}=\frac{\phi_{i}-\phi_{\max , i}}{\left|\phi_{\min , i}\right|} \leq 0 \quad \text { on } \Gamma_{C} \quad \mathrm{i}=1,2, \ldots, \mathrm{n} \\
g_{j}=\frac{\phi_{\min , j}-\phi_{j}}{\left|\phi_{\min , j}\right|} \leq 0 \quad \text { on } \Gamma_{C} \quad \mathrm{j}=1,2, \ldots, \mathrm{n}
\end{gathered}
$$

where

$\mathrm{n}_{\mathrm{s}}$ is the number of surfaces

$\mathrm{n}_{\mathrm{e}}$ is the number of elements per surface

$\varphi_{\text {target }}$ is the target potential per surface

$\phi_{\max , j}$ and $\phi_{\min , j}$ are the max and min protection potential respectively.

\subsection{Magnetic fields}

The CRM signature generated by the currents following in the sea water was calculated by solving the vector potential [1] using current data from the BEM: 


$$
\begin{array}{cl}
\qquad \nabla^{2} A=-\mu J \\
\text { where } \quad \mathrm{A}=\text { vector potential } \\
\mathrm{J}=\text { vector of current density components }
\end{array}
$$

The magnetic field is then simply $\mathrm{B}=\operatorname{curl} \mathrm{A}$.

\section{Results and discussion}

\subsection{The performance of initial ICCP system on shipboard}

On the vessel single zone four anodes ICCP system was used to protect the hull and appendages. The anodes were symmetrically installed at $142^{\text {nd }}$ frame and $242^{\text {nd }}$ frame and the initial input current was $8 \mathrm{~A}$ and $10 \mathrm{~A}$ respectively. SSE reference electrode was installed at $186^{\text {th }}$ frame. When the reference electrode read of $-850 \mathrm{mV}$ (vs. SSE), the potential distribution on the hull was shown in Figure 3. The calculation results indicated that the hull, propellers and rudders were under protect, the range in potential of hull was from $-846 \mathrm{mV}$ to $-1000 \mathrm{mV}$. The current output of anode at $142^{\text {nd }}$ frame and $242^{\text {nd }}$ frame was $5.74 \mathrm{~A}$ and $7.40 \mathrm{~A}$ respectively.

At the same time, the practical ship protective potential of $37^{\text {th }}, 72^{\text {nd }}, 128^{\text {th }}$, $168^{\text {th }}$ and $228^{\text {th }}$ frame was measured when the ship berthed at port by $\mathrm{Cu} / \mathrm{CuSO}_{4}$ reference electrode. Compared with the measured results, the calculation results showed the same trend as the practical measured data and were near the same values as the measured results, as shown in figure 4 . That is to say the calculation results are accurate.

The CRM field distribution on the sea level was shown in figure 5, where $\mathrm{x}$ axis was parallel with the keel of ship and y axis was vertical to the keel. The keel of the ship was at the position of $y=0 \mathrm{~m}$. The calculation results indicated that the most strength magnetic field position was the region around $142^{\text {nd }}$ frame where anodes were installed and then was the position of stern around $240^{\text {th }}$ frame, while the normal of the magnetic field at the two positions was inverted. In figure 5, figure 7 and figure 9, the same color denoted the same strength of the magnetic field and ever color represented the same gradient of magnetic field.

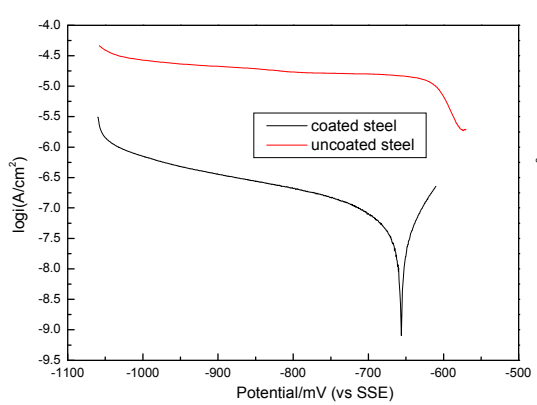

(a) carbon steel

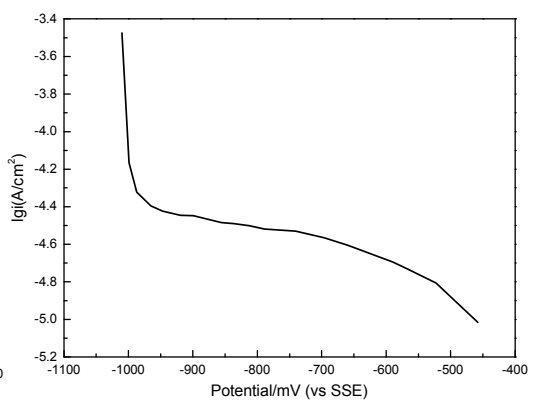

(b) NAB

Figure 2: Boundary condition. 


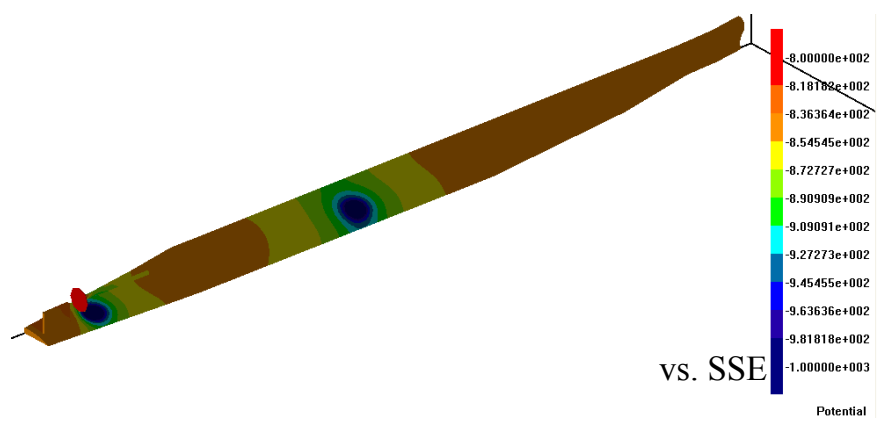

Figure 3: Potential plot of vessel protected by the initial ICCP system.

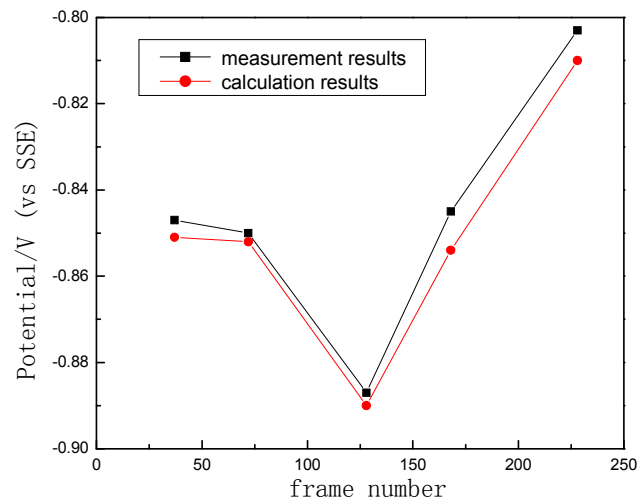

Figure 4: $\quad$ Potential vs. frame.

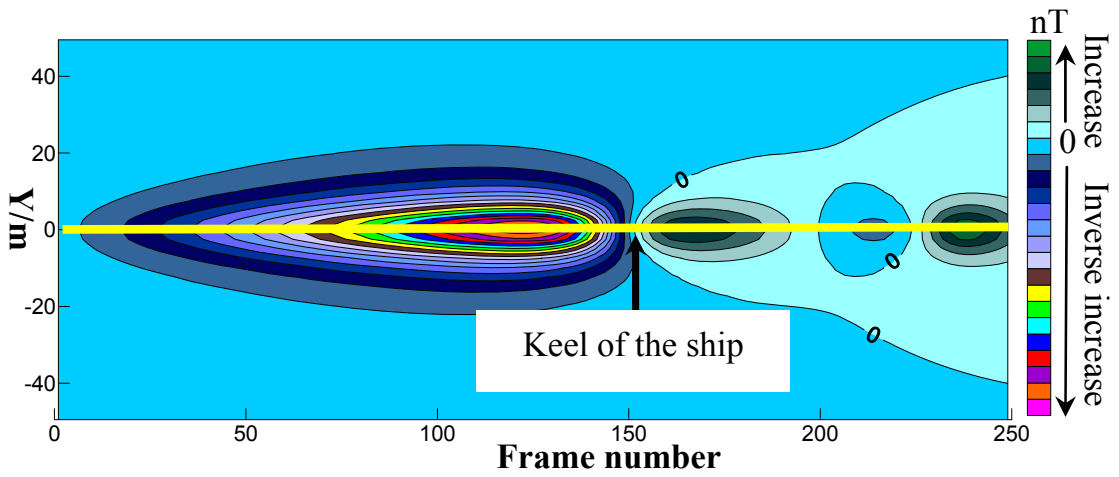

Figure 5: $\quad$ CRM field generated by the initial ICCP system. 


\subsection{ICCP system optimization}

\subsubsection{Anodes position optimization}

Based on experientially designed ICCP system, one group of anodes was set at $232^{\text {th }}$ because the propellers consume more current. The other group of anodes was initially set at $115^{\text {th }}$ frame, the candidate positions were set at $115^{\text {th }}, 120^{\text {th }}$, $130^{\text {th }}, 135^{\text {th }}, 142^{\text {nd }}$ and $150^{\text {th }}$ frame. By comparing the potential and magnetic field distribution of different anodes setting ICCP system, the best ICCP system was sought, which anodes were installed at $130^{\text {th }}$ frame and $232^{\text {nd }}$ frame. When the initial input current of framel and frame 2 was $5.7 \mathrm{~A}$ and $7.4 \mathrm{~A}$ respectively and the reference electrode read of $-850 \mathrm{mV}$ vs. SSE, the potential distribution of ship hull was shown in figure 6 , which ranged from $-821 \mathrm{mV}$ to $-1000 \mathrm{mV}$. The current output of anode at $130^{\text {nd }}$ frame and $232^{\text {nd }}$ frame was $5.32 \mathrm{~A}$ and $7.51 \mathrm{~A}$ respectively.

After optimization, the CRM field distribution on sea level was shown in figure 7 . In figure 7, we can know that the most strength magnetic field position changed to $130^{\text {th }}$ frame, the relative strong magnetic field position changed to $160^{\text {th }}$ frame. Compared with the CRM distribution of initial ICCP system, by moving the position of anode, although the CRM field strength increased at stern, the magnetic field around ship was improved as shown in figure 8, which show the magnetic field distribution at $\mathrm{y}=0$ from bow to stern. The magnetic signature increase between $150^{\text {th }}$ frame and $200^{\text {th }}$ frame may be related to the distance increase between the two group anodes. The distance increase resulted in the current density increase between them, which led to the magnetic signature increased. By anode position optimization, the magnetic field strength was reduced about $30 \%$.

\subsubsection{Anodes output current optimization}

Once the position of anode was fixed, the output current of anodes optimization was carried out. The purpose of current optimized was to minimize the corrosion

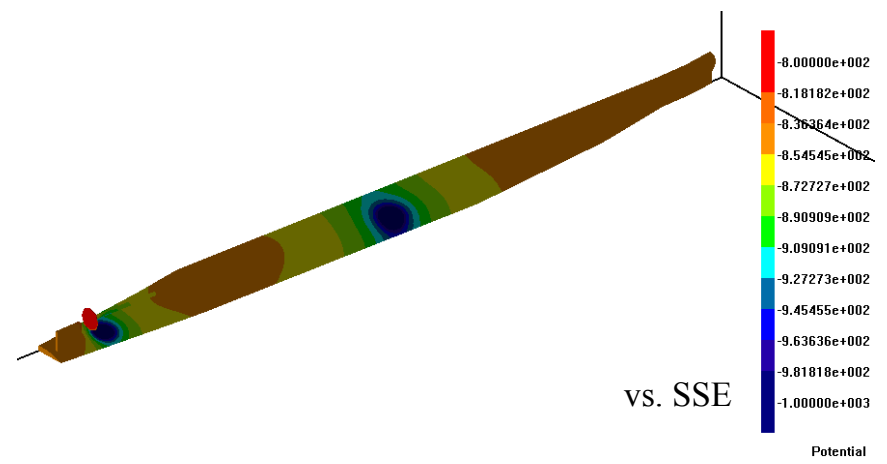

Figure 6: $\quad$ Potential plot of the vessel protected by the optimized anodes position ICCP system. 


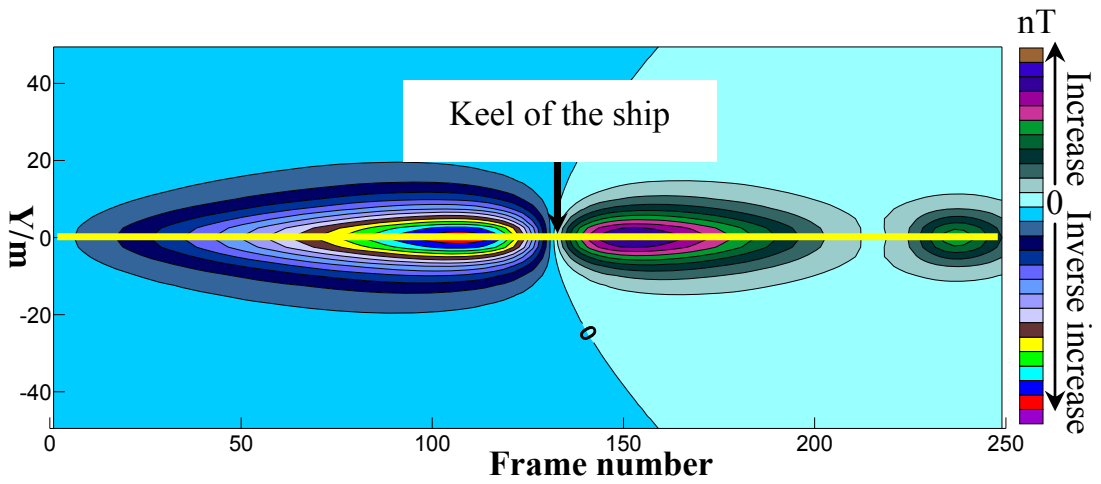

Figure 7: $\quad$ CRM field distribution after anodes position optimization.

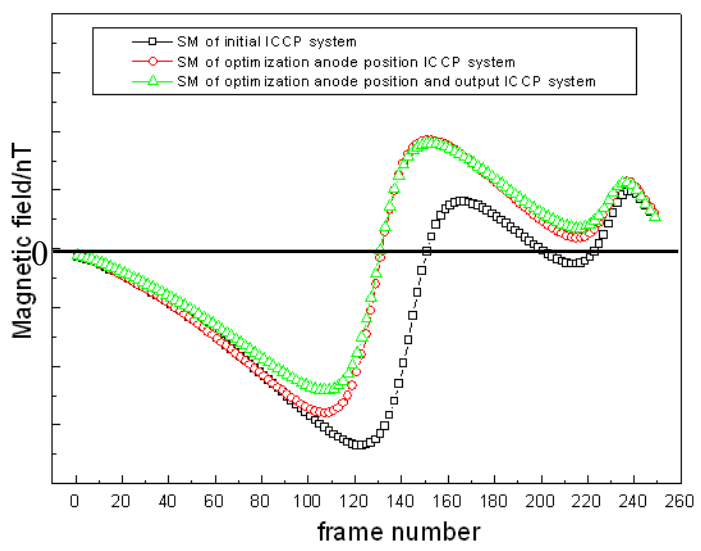

Figure 8: $\quad$ CRM field distribution at $\mathrm{y}=0 \mathrm{~m}$.

related electromagnetic signature while the hull obtained sufficient $\mathrm{CP}$ effect. The initial input current of frame 1 and frame 2 was $5.3 \mathrm{~A}$ and $7.5 \mathrm{~A}$ respectively. After current optimization, the potential distribution on the hull was shown in figure 9. Compared with figure 3 and figure 5, the protective potential obviously changed more positive after current optimization, but the protective potential lower than $-800 \mathrm{mV}$, while the current output of anodes at $130^{\text {th }}$ frame and $232^{\text {nd }}$ minimized to $5.08 \mathrm{~A}$ and $6.93 \mathrm{~A}$ respectively. Thus by anode position and output current optimization, the output current was reduced $8.6 \%$.

The magnetic field was further reduced after current optimization as shown in figure 10. Compared with the magnetic field generated by initial ICCP system, the strength of magnetic was reduced about $40 \%$. Like the magnetic field of anode position optimized ICCP system, the magnetic field around the position where anode was installed was most strong. In order to more intuitively exhibit the magnetic field distribution of initial and optimized ICCP systems, the 


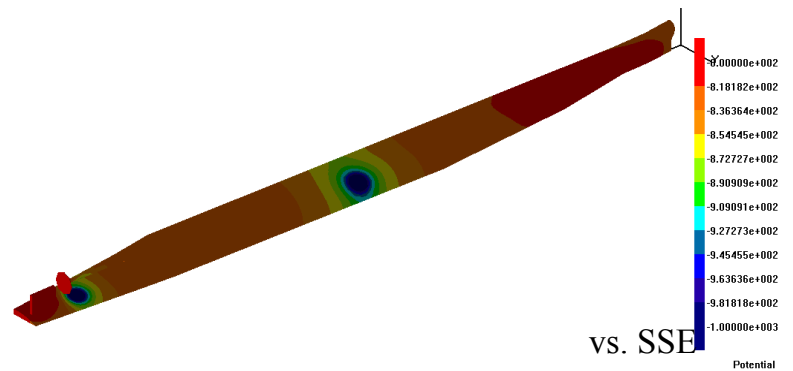

Figure 9: Potential distribution after anodes position and output current optimization.

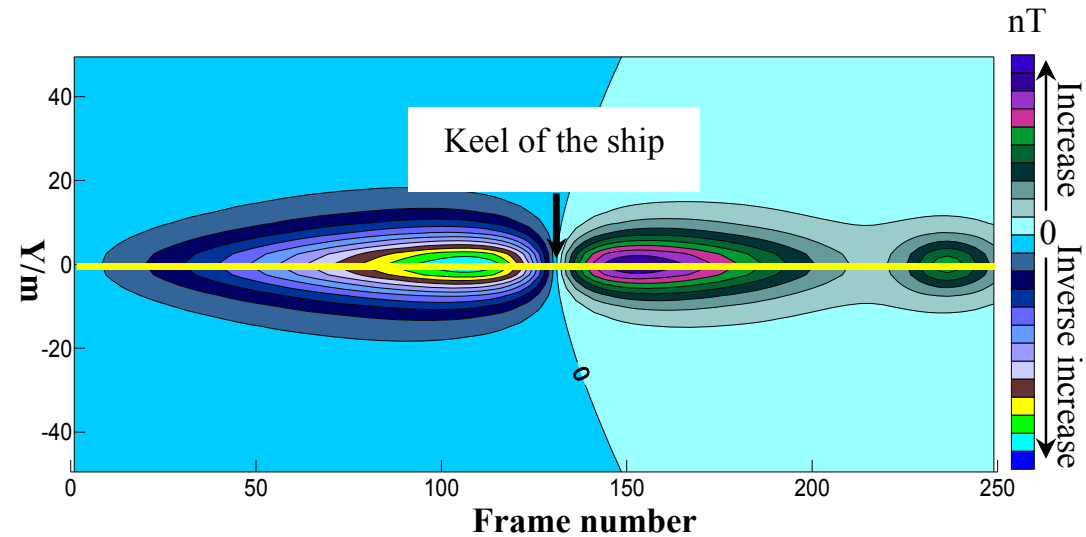

Figure 10: CRM field distribution after anodes position and current optimization.

magnetic signature at $\mathrm{y}=0$ was shown in figure 8 . In this figure, we can distinctly know that the magnetic field around ship was step by step reduced by position and current of anodes optimization.

\section{Conclusions}

The BEM can reasonably predict and optimize the potential distribution and provide data to predict and minimize the magnetic signature of a vessel.

Anode position optimization is an effective approach to control CRM signature, for the ship we studied, when the anodes were symmetrically installed at $130^{\text {th }}$ frame and $232^{\text {nd }}$ frame, the signature was least.

The magnetic signature was reduced about twenty-five percent by the anode position and output current optimization. 


\section{References}

[1] E. Santana. Diaz, R. Adey, J. Baynham et.al, Optimisation of ICCP systems to minimise electric signatures, www.beasy.com/publications

[2] R. Adey and J. Baynham, Predicting corrosion related electrical and magnetic fields using BEM, www.beasy.com/publications

[3] E. Santana Diaz and R. Adey, Optimisation of the performance of an ICCP system by changing current supplied and position of the anode, Boundary Elements XXIV, pp. 1-16.2003

[4] E. S. Diaz, A Complete Underwater Electric and Magnetic Signature Scenario Using Computational Modelling, www.beasy.com/publications

[5] E. Santana Diza and R. Adey, Optimising the location of anodes in cathodic protection systems to smooth potential distribution, Advances in Engineering Software, 36, pp. 592, 2005

[6] E. Santana Diza and R. Adey, Predicting the coating condition on ships using ICCP system data, www.beasy.com/publications 